\section{CRYSTALLOGRAPHIC COMMUNICATIONS}

ISSN 2056-9890

\section{Crystal structure of 4,5-dinitro- $1 \mathrm{H}$ - imidazole}

\author{
G. Kenneth Windler, ${ }^{a}$ Brian L. Scott, ${ }^{b}$ Neil C. Tomson ${ }^{b}$ \\ and Philip W. Leonard ${ }^{\mathrm{a} *}$
}

${ }^{\text {a} P O ~ B o x ~} 1663$ MS C920, Los Alamos National Laboratory, Los Alamos, NM 87544, USA, and ${ }^{\mathbf{b}} \mathrm{PO}$ Box 1663 MS J514, Los Alamos National Laboratory, Los Alamos, NM 87544, USA. *Correspondence e-mail: philipl@lanl.gov

Received 6 June 2015; accepted 13 July 2015

Edited by G. Smith, Queensland University of Technology, Australia

The title compound, $\mathrm{C}_{3} \mathrm{H}_{2} \mathrm{~N}_{4} \mathrm{O}_{4}$, forms crystals with two molecules in the asymmetric unit which are conformationally similar. With the exception of the $\mathrm{O}$ atoms of the nitro groups, the molecules are essentially planar. In the crystal, adjacent molecules are associated by $\mathrm{N}-\mathrm{H} \cdots \mathrm{N}$ hydrogen bonds involving the imidazole $\mathrm{N}-\mathrm{H}$ donors and $\mathrm{N}$-atom acceptors of the unsaturated nitrogen of neighboring rings, forming layers parallel to (010).

Keywords: crystal structure; 4,5-dinitro-1 $\mathrm{H}$-imidazole; hydrogen bonding.

CCDC reference: 1412685

\section{Related literature}

For background to imidazoles and the title compound, see: Windaus \& Vogt (1907); Cooper (1996); Epishina et al. (1967). For the preparation, see: Novikov et al. (1970). For similar structures, see: Parrish et al. (2015); Windler et al. (2015).

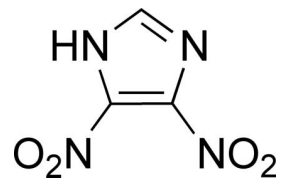

\section{Experimental}

\subsection{Crystal data}

$\mathrm{C}_{3} \mathrm{H}_{2} \mathrm{~N}_{4} \mathrm{O}_{4}$

$M_{r}=158.09$

Monoclinic, $P 2_{1} / n$

$a=11.4797$ (9) $\AA$

$b=8.8205$ (7) $\AA$

$c=11.802(1) \AA$

$\beta=107.827$ (1) ${ }^{\circ}$

$$
\begin{aligned}
& V=1137.65(16) \AA^{3} \\
& Z=8 \\
& \text { Mo } K \alpha \text { radiation } \\
& \mu=0.17 \mathrm{~mm}^{-1} \\
& T=100 \mathrm{~K} \\
& 0.12 \times 0.06 \times 0.06 \mathrm{~mm}
\end{aligned}
$$

\subsection{Data collection \\ Bruker D8 Quest with CMOS diffractometer \\ Absorption correction: multi-scan (SADABS; Bruker, 2009) \\ 25837 measured reflections 4868 independent reflections 4216 reflections with $I>2 \sigma(I)$ $R_{\text {int }}=0.024$}

$T_{\min }=0.971, T_{\max }=0.995$

\subsection{Refinement}

$R\left[F^{2}>2 \sigma\left(F^{2}\right)\right]=0.037$

$w R\left(F^{2}\right)=0.118$

$S=1.60$

4868 reflections

\author{
211 parameters \\ All $\mathrm{H}$-atom parameters refined \\ $\Delta \rho_{\max }=0.54 \mathrm{e} \AA^{-3}$ \\ $\Delta \rho_{\min }=-0.33 \mathrm{e}^{-3}$
}

Table 1

Hydrogen-bond geometry $\left(\AA{ }^{\circ}\right)$.

\begin{tabular}{lllll}
\hline$D-\mathrm{H} \cdots A$ & $D-\mathrm{H}$ & $\mathrm{H} \cdots A$ & $D \cdots A$ & $D-\mathrm{H} \cdots A$ \\
\hline $\mathrm{N} 3-\mathrm{H} 2 \cdots \mathrm{N} 7^{\mathrm{i}}$ & $0.90(2)$ & $1.96(2)$ & $2.836(1)$ & $163(2)$ \\
$\mathrm{N} 8-\mathrm{H} 4 \cdots \mathrm{N} 4^{\text {ii }}$ & $0.92(2)$ & $1.89(2)$ & $2.807(1)$ & $179(3)$ \\
\hline
\end{tabular}

Symmetry codes: (i) $-x+\frac{1}{2}, y-\frac{1}{2},-z+\frac{1}{2}$; (ii) $-x,-y+1,-z+1$.

Data collection: APEX2 (Bruker, 2009); cell refinement: SAINT (Bruker, 2009); data reduction: $S A I N T$; program(s) used to solve structure: SHELXS97 (Sheldrick, 2008); program(s) used to refine structure: SHELXL97 (Sheldrick, 2008); molecular graphics: ORTEP-3 for Windows (Farrugia, 2012), Mercury (Macrae et al., 2008) and PLATON (Spek, 2009); software used to prepare material for publication: CHEMDRAW Ultra (Cambridge Soft, 2014).

\section{Acknowledgements}

This work was supported by the National Nuclear Security Administration Science Campaign 2 and performed at Los Alamos National Laboratory under DE-AC52-06 N A25396. LA-UR-15-23929

Supporting information for this paper is available from the $\mathrm{IUCr}$ electronic archives (Reference: ZS2338).

\section{References}

Bruker (2009). APEX2, SAINT and $S A D A B S$. Bruker AXS Inc., Madison, Wisconsin, USA.

Cambridge Soft (2014). CHEMDRAW Ultra. Cambridge Soft Corporation, Cambridge, Massachusetts, USA.

Cooper, P. W. (1996). In Explosives Engineering. New York: Wiley-VCH.

Epishina, L. V., Slovetskii, V. I., Osipov, V. G., Lebedev, O. V., Khmel'nitskii, L. I., Sevost'yanova, V. V. \& Novikova, T. S. (1967). Khim. Geterotsikl. Soedin. 4, 716-723.

Farrugia, L. J. (2012). J. Appl. Cryst. 45, 849-854.

Macrae, C. F., Bruno, I. J., Chisholm, J. A., Edgington, P. R., McCabe, P., Pidcock, E., Rodriguez-Monge, L., Taylor, R., van de Streek, J. \& Wood, P. A. (2008). J. Appl. Cryst. 41, 466-470.

Novikov, S. S., Khmel'nitskii, L. I., Lebedev, O. V., Epishina, L. V. \& Sevost'yanova, V. V. (1970). Khim. Geterotsikl. Soedin. 5, 664-668.

Parrish, D. A., Kramer, S., Windler, G. K., Chavez, D. E. \& Leonard, P. W. (2015). Acta Cryst. E71, o491.

Sheldrick, G. M. (2008). Acta Cryst. A64, 112-122.

Spek, A. L. (2009). Acta Cryst. D65, 148-155.

Windaus, A. \& Vogt, W. (1907). Ber. Dtsch. Chem. Ges. 40, 3691-3695.

Windler, G. K., Scott, B. L., Tomson, N. C. \& Leonard, P. W. (2015). Acta Cryst. E71, o633. 


\section{supporting information}

Acta Cryst. (2015). E71, o634 [https://doi.org/10.1107/S2056989015013432]

\section{Crystal structure of 4,5-dinitro-1H-imidazole}

\section{G. Kenneth Windler, Brian L. Scott, Neil C. Tomson and Philip W. Leonard}

\section{S1. Comment}

In addition to more mundane uses as pharmaceuticals (Windaus \& Vogt, 1907), imidazoles make quality backbones for energetic materials (Epishina et al., 1967) because of their nitrogen content. The dinitro-bearing title compound, $\mathrm{C}_{3} \mathrm{H}_{2} \mathrm{~N}_{4} \mathrm{O}_{4}$, is of interest because of its better oxygen balance (Cooper, 1996), contributing to its effectiveness as an explosive. To better understand the nature of explosive sensitivity as it relates to intermolecular forces, the title compound (Fig. 1) was of interest for comparison with other imidazoles previously studied (Parrish et al., 2015; Windler et al., 2015).

In the title compound, the two independent molecules ( $A$, defined by $\mathrm{C} 1-\mathrm{N} 3$ and $B$, defined by $\mathrm{C} 4-\mathrm{N} 7)$ in the asymmetric unit (Fig. 1) are conformationally similar with the nitro groups being variously rotated out of the imidazole planes: in $A$ [torsion angles $\mathrm{N} 3-\mathrm{C} 1-\mathrm{N} 1-\mathrm{O} 2,-174.29(9)^{\circ}$ and $\mathrm{N} 4-\mathrm{C} 3-\mathrm{N} 2-\mathrm{O} 3,163.63(7)^{\circ}$ ] and in $B$ [torsion angles $\mathrm{N} 7-\mathrm{C} 4-\mathrm{N} 5-\mathrm{O} 6,156.95(8)^{\circ}$ and $\left.\mathrm{N} 6-\mathrm{C} 6-\mathrm{N} 6-\mathrm{O} 7,163.63(7)^{\circ}\right]$.

In the crystal, intermolecular $\mathrm{N}-\mathrm{H} \cdots \mathrm{N}$ hydrogen bonding interactions $\mathrm{N} 3-\mathrm{H} \cdots \mathrm{N} 7$ and $\mathrm{N} 8-\mathrm{H} \cdots \mathrm{N} 4$ between the $A$ and $B$ molecules (Table 1), generate layered structures lying roughly parallel to (010) (Fig. 2).

\section{S2. Experimental}

Caution! The title compound is an explosive and should only be handled with appropriate safety equipment in small quantities by an experienced explosive handler.

The title compound was prepared by literature methods (Novikov et al., 1970). Crystals were obtained by slow evaporation of a concentrated solution in ethyl acetate.

\section{S3. Refinement}

All hydrogen atoms was located in a difference-Fourier and the positional parameters were fully refined, with $U_{\text {iso }}(\mathrm{H})$ set invariant at 0.08 . 

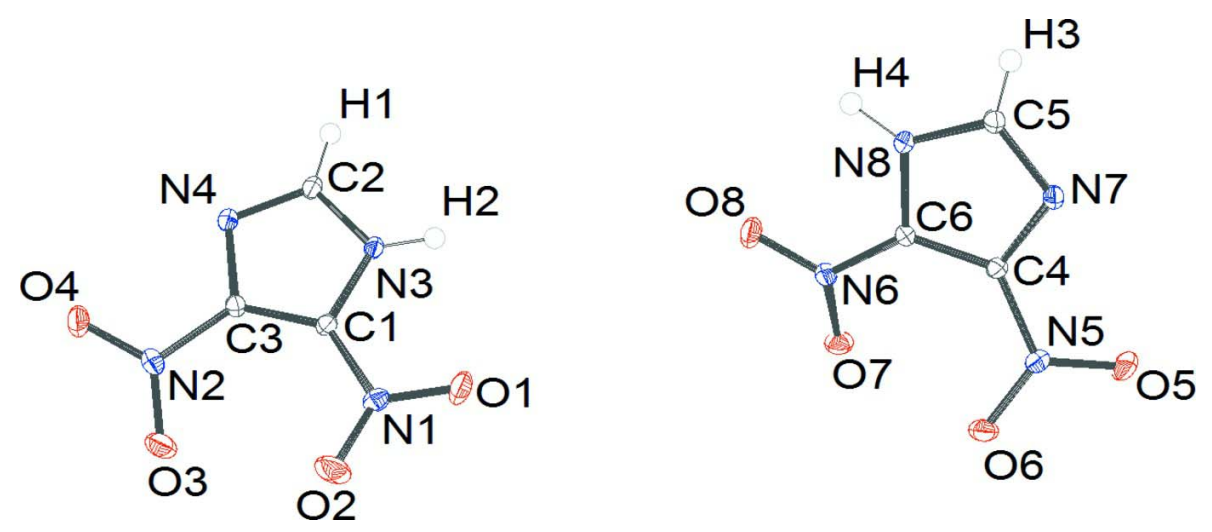

Figure 1

The molecular structure of the title compound with atom labeling. Ellipsoids are drawn at the $50 \%$ probability level and the hydrogen atoms are drawn as spheres of arbitrary size.

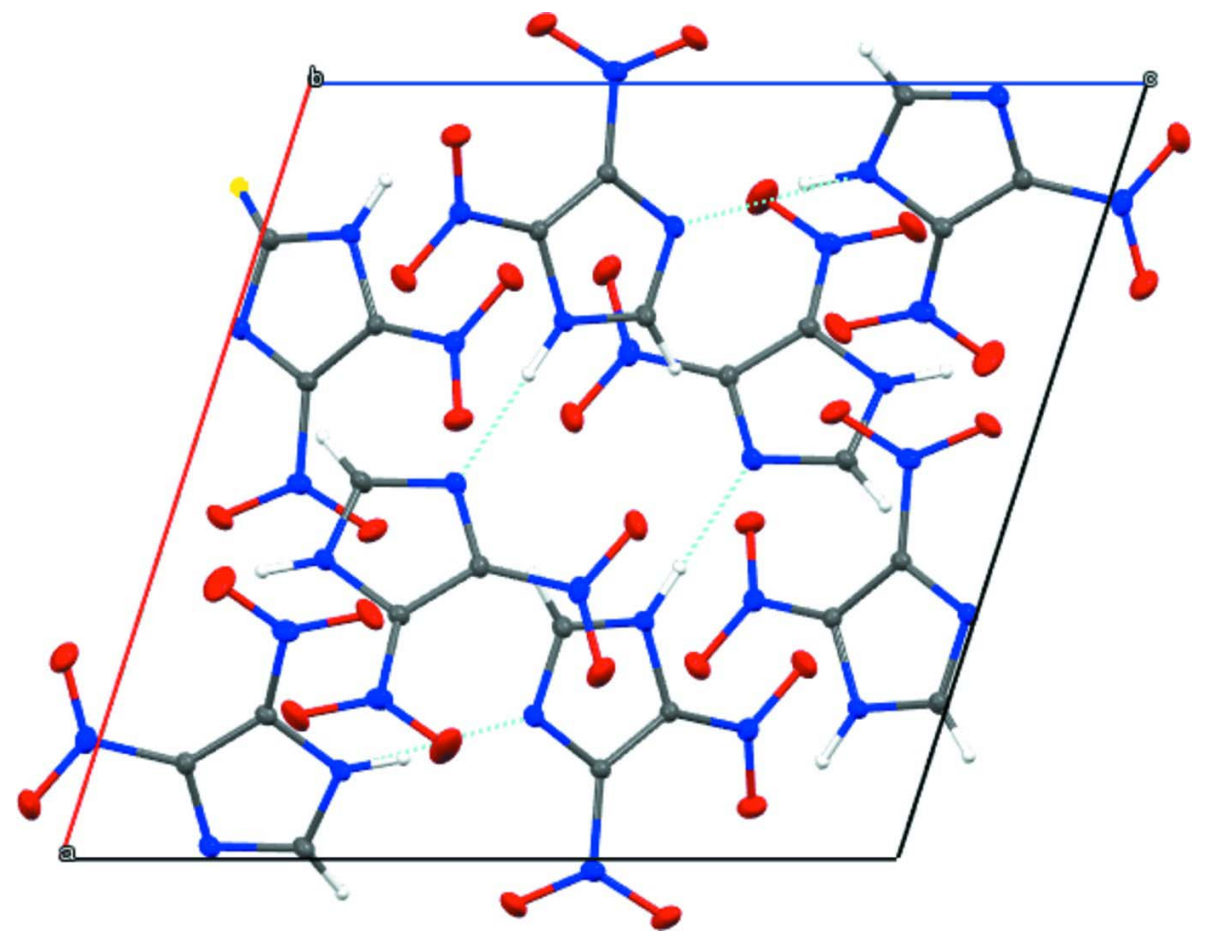

Figure 2

A crystal packing diagram for the title compound viewed along the $b$ axis. The $\mathrm{N}-\mathrm{H} \cdots \mathrm{N}$ hydrogen bonds are shown as dashed lines.

\section{4,5-Dinitro-1H-imidazole}

\section{Crystal data}

$\mathrm{C}_{3} \mathrm{H}_{2} \mathrm{~N}_{4} \mathrm{O}_{4}$

$M_{r}=158.09$

Monoclinic, $P 2{ }_{1} / n$

Hall symbol: -P 2 yn

$a=11.4797$ (9) $\AA$

$b=8.8205$ (7) $\AA$

$$
\begin{aligned}
& c=11.802(1) \AA \\
& \beta=107.827(1)^{\circ} \\
& V=1137.65(16) \AA^{3} \\
& Z=8 \\
& F(000)=640 \\
& D_{\mathrm{x}}=1.846 \mathrm{Mg} \mathrm{m}^{-3}
\end{aligned}
$$


Melting point $=460-461 \mathrm{~K}$

Mo $K \alpha$ radiation, $\lambda=0.71073 \AA$

Cell parameters from 4868 reflections

$\theta=2.9-35.4^{\circ}$

\section{Data collection}

Bruker D8 Quest with CMOS diffractometer

Radiation source: fine-focus sealed tube

Bruker Triumph curved graphite

monochromator

$\omega$ scans

Absorption correction: multi-scan

(SADABS; Bruker, 2009)

$T_{\min }=0.971, T_{\max }=0.995$

\section{Refinement}

Refinement on $F^{2}$

Least-squares matrix: full

$R\left[F^{2}>2 \sigma\left(F^{2}\right)\right]=0.037$

$w R\left(F^{2}\right)=0.118$

$S=1.60$

4868 reflections

211 parameters

0 restraints

Primary atom site location: structure-invariant direct methods

$$
\begin{aligned}
& \mu=0.17 \mathrm{~mm}^{-1} \\
& T=100 \mathrm{~K} \\
& \text { Block, colorless } \\
& 0.12 \times 0.06 \times 0.06 \mathrm{~mm}
\end{aligned}
$$

25837 measured reflections

4868 independent reflections

4216 reflections with $I>2 \sigma(I)$

$R_{\text {int }}=0.024$

$\theta_{\max }=35.4^{\circ}, \theta_{\min }=2.9^{\circ}$

$h=-17 \rightarrow 18$

$k=-14 \rightarrow 13$

$l=-18 \rightarrow 18$

Secondary atom site location: difference Fourier map

Hydrogen site location: inferred from neighbouring sites

All $\mathrm{H}$-atom parameters refined

$w=1 /\left[\sigma^{2}\left(F_{0}^{2}\right)+(0.0548 P)^{2}\right]$

where $P=\left(F_{\mathrm{o}}^{2}+2 F_{\mathrm{c}}{ }^{2}\right) / 3$

$(\Delta / \sigma)_{\max }=0.001$

$\Delta \rho_{\max }=0.54$ e $\AA^{-3}$

$\Delta \rho_{\min }=-0.33$ e $\AA^{-3}$

Special details

Geometry. All e.s.d.'s (except the e.s.d. in the dihedral angle between two 1.s. planes) are estimated using the full covariance matrix. The cell e.s.d.'s are taken into account individually in the estimation of e.s.d.'s in distances, angles and torsion angles; correlations between e.s.d.'s in cell parameters are only used when they are defined by crystal symmetry. An approximate (isotropic) treatment of cell e.s.d.'s is used for estimating e.s.d.'s involving 1.s. planes.

Refinement. Refinement of $F^{2}$ against ALL reflections. The weighted $R$-factor $w R$ and goodness of fit $S$ are based on $F^{2}$, conventional $R$-factors $R$ are based on $F$, with $F$ set to zero for negative $F^{2}$. The threshold expression of $F^{2}>\sigma\left(F^{2}\right)$ is used only for calculating $R$-factors(gt) etc. and is not relevant to the choice of reflections for refinement. $R$-factors based on $F^{2}$ are statistically about twice as large as those based on $F$, and $R$ - factors based on ALL data will be even larger.

Fractional atomic coordinates and isotropic or equivalent isotropic displacement parameters $\left(\AA^{2}\right)$

\begin{tabular}{lllll}
\hline & $x$ & $y$ & $z$ & $U_{\text {iso }} * / U_{\text {eq }}$ \\
\hline $\mathrm{N} 1$ & $0.29370(6)$ & $0.50168(8)$ & $0.81909(6)$ & $0.01298(13)$ \\
$\mathrm{N} 2$ & $0.15331(6)$ & $0.38337(8)$ & $1.01582(6)$ & $0.01315(13)$ \\
$\mathrm{N} 3$ & $0.11402(6)$ & $0.37089(8)$ & $0.70000(6)$ & $0.01075(12)$ \\
$\mathrm{N} 4$ & $0.01562(6)$ & $0.30341(8)$ & $0.82719(6)$ & $0.01203(13)$ \\
$\mathrm{N} 5$ & $0.51594(6)$ & $0.79131(8)$ & $0.14048(6)$ & $0.01209(13)$ \\
$\mathrm{N} 6$ & $0.33377(6)$ & $0.62492(8)$ & $0.27135(6)$ & $0.01158(12)$ \\
$\mathrm{N} 7$ & $0.31591(6)$ & $0.85813(8)$ & $0.01033(6)$ & $0.01174(12)$ \\
$\mathrm{N} 8$ & $0.19372(6)$ & $0.75974(8)$ & $0.10560(6)$ & $0.01083(12)$ \\
O1 & $0.31536(6)$ & $0.52596(8)$ & $0.72497(6)$ & $0.02044(14)$ \\
O2 & $0.35443(7)$ & $0.54920(9)$ & $0.91643(6)$ & $0.02736(17)$ \\
O3 & $0.25949(6)$ & $0.41102(8)$ & $1.07370(6)$ & $0.01982(14)$
\end{tabular}




$\begin{array}{lllll}\text { O4 } & 0.06957(6) & 0.36304(9) & 1.05882(6) & 0.02084(14) \\ \text { O5 } & 0.55956(6) & 0.80860(9) & 0.05851(6) & 0.02079(14) \\ \text { O6 } & 0.57642(6) & 0.78190(8) & 0.24607(5) & 0.01798(13) \\ \text { O7 } & 0.43309(5) & 0.56137(7) & 0.30593(6) & 0.01585(12) \\ \text { O8 } & 0.24913(6) & 0.60769(8) & 0.31361(6) & 0.01785(13) \\ \text { C1 } & 0.18544(7) & 0.41388(8) & 0.81011(6) & 0.01016(13) \\ \text { C2 } & 0.01380(7) & 0.30381(9) & 0.71412(7) & 0.01240(14) \\ \text { C3 } & 0.12271(7) & 0.37018(8) & 0.88769(6) & 0.01062(13) \\ \text { C4 } & 0.38434(7) & 0.78740(8) & 0.11035(6) & 0.01003(13) \\ \text { C5 } & 0.20090(7) & 0.83961(9) & 0.01039(7) & 0.01204(14) \\ \text { C6 } & 0.31069(7) & 0.72465(8) & 0.17067(6) & 0.00992(13) \\ \text { H1 } & -0.044(2) & 0.259(3) & 0.651(2) & 0.080^{*} \\ \text { H2 } & 0.129(2) & 0.386(3) & 0.630(2) & 0.080^{*} \\ \text { H3 } & 0.133(2) & 0.875(3) & -0.045(2) & 0.080^{*} \\ \text { H4 } & 0.125(2) & 0.740(3) & 0.1274(19) & 0.080^{*}\end{array}$

Atomic displacement parameters $\left(\AA^{2}\right)$

\begin{tabular}{lllllll}
\hline & $U^{11}$ & $U^{22}$ & $U^{33}$ & $U^{12}$ & $U^{13}$ & $U^{23}$ \\
\hline $\mathrm{N} 1$ & $0.0108(3)$ & $0.0118(3)$ & $0.0163(3)$ & $-0.0018(2)$ & $0.0041(2)$ & $-0.0016(2)$ \\
$\mathrm{N} 2$ & $0.0163(3)$ & $0.0130(3)$ & $0.0101(3)$ & $0.0017(2)$ & $0.0039(2)$ & $0.0008(2)$ \\
$\mathrm{N} 3$ & $0.0099(3)$ & $0.0133(3)$ & $0.0102(3)$ & $-0.0015(2)$ & $0.0047(2)$ & $-0.0021(2)$ \\
$\mathrm{N} 4$ & $0.0110(3)$ & $0.0151(3)$ & $0.0109(3)$ & $-0.0013(2)$ & $0.0047(2)$ & $-0.0008(2)$ \\
$\mathrm{N} 5$ & $0.0099(3)$ & $0.0128(3)$ & $0.0138(3)$ & $-0.0012(2)$ & $0.0039(2)$ & $0.0000(2)$ \\
$\mathrm{N} 6$ & $0.0119(3)$ & $0.0126(3)$ & $0.0100(3)$ & $-0.0010(2)$ & $0.0031(2)$ & $0.0009(2)$ \\
$\mathrm{N} 7$ & $0.0108(3)$ & $0.0154(3)$ & $0.0098(3)$ & $0.0015(2)$ & $0.0042(2)$ & $0.0018(2)$ \\
$\mathrm{N} 8$ & $0.0089(3)$ & $0.0147(3)$ & $0.0096(3)$ & $0.0004(2)$ & $0.0038(2)$ & $0.0005(2)$ \\
O1 & $0.0205(3)$ & $0.0243(3)$ & $0.0221(3)$ & $-0.0084(2)$ & $0.0147(2)$ & $-0.0065(2)$ \\
O2 & $0.0271(4)$ & $0.0329(4)$ & $0.0170(3)$ & $-0.0173(3)$ & $-0.0009(3)$ & $-0.0019(3)$ \\
O3 & $0.0195(3)$ & $0.0229(3)$ & $0.0135(3)$ & $-0.0047(2)$ & $-0.0003(2)$ & $-0.0017(2)$ \\
O4 & $0.0197(3)$ & $0.0315(4)$ & $0.0139(3)$ & $0.0045(3)$ & $0.0091(2)$ & $0.0044(2)$ \\
O5 & $0.0135(3)$ & $0.0340(4)$ & $0.0182(3)$ & $-0.0032(2)$ & $0.0097(2)$ & $-0.0037(2)$ \\
O6 & $0.0123(3)$ & $0.0222(3)$ & $0.0156(3)$ & $-0.0039(2)$ & $-0.0014(2)$ & $0.0056(2)$ \\
O7 & $0.0114(2)$ & $0.0170(3)$ & $0.0171(3)$ & $0.0014(2)$ & $0.0013(2)$ & $0.0044(2)$ \\
O8 & $0.0166(3)$ & $0.0229(3)$ & $0.0173(3)$ & $0.0010(2)$ & $0.0099(2)$ & $0.0062(2)$ \\
C1 & $0.0093(3)$ & $0.0104(3)$ & $0.0113(3)$ & $-0.0006(2)$ & $0.0039(2)$ & $-0.0013(2)$ \\
C2 & $0.0105(3)$ & $0.0162(3)$ & $0.0118(3)$ & $-0.0022(2)$ & $0.0053(2)$ & $-0.0021(2)$ \\
C3 & $0.0111(3)$ & $0.0118(3)$ & $0.0093(3)$ & $0.0004(2)$ & $0.0037(2)$ & $-0.0004(2)$ \\
C4 & $0.0086(3)$ & $0.0120(3)$ & $0.0097(3)$ & $0.0000(2)$ & $0.0031(2)$ & $-0.0006(2)$ \\
C5 & $0.0109(3)$ & $0.0157(3)$ & $0.0100(3)$ & $0.0021(2)$ & $0.0040(2)$ & $0.0016(2)$ \\
C6 & $0.0095(3)$ & $0.0121(3)$ & $0.0080(3)$ & $0.0000(2)$ & $0.0025(2)$ & $0.0005(2)$ \\
& & & & & & \\
\hline & & & & & & \\
\hline
\end{tabular}

Geometric parameters $\left(A,{ }^{o}\right)$

\begin{tabular}{llll}
\hline $\mathrm{O} 1-\mathrm{N} 1$ & $1.2291(10)$ & $\mathrm{N} 4-\mathrm{C} 3$ & $1.3530(11)$ \\
$\mathrm{O} 2-\mathrm{N} 1$ & $1.2211(10)$ & $\mathrm{N} 3-\mathrm{H} 2$ & $0.90(2)$ \\
$\mathrm{O} 3-\mathrm{N} 2$ & $1.2256(10)$ & $\mathrm{N} 5-\mathrm{C} 4$ & $1.4426(11)$ \\
$\mathrm{O} 4-\mathrm{N} 2$ & $1.2299(10)$ & $\mathrm{N} 6-\mathrm{C} 6$ & $1.4364(10)$
\end{tabular}




\begin{tabular}{|c|c|c|c|}
\hline $\mathrm{O} 5-\mathrm{N} 5$ & $1.2274(10)$ & $\mathrm{N} 7-\mathrm{C} 5$ & $1.3306(11)$ \\
\hline $\mathrm{O} 6-\mathrm{N} 5$ & $1.2297(9)$ & $\mathrm{N} 7-\mathrm{C} 4$ & $1.3535(10)$ \\
\hline $\mathrm{O} 7-\mathrm{N} 6$ & $1.2230(10)$ & $\mathrm{N} 8-\mathrm{C} 6$ & $1.3628(11)$ \\
\hline $\mathrm{O} 8-\mathrm{N} 6$ & $1.2299(10)$ & $\mathrm{N} 8-\mathrm{C} 5$ & $1.3500(11)$ \\
\hline $\mathrm{N} 1-\mathrm{C} 1$ & $1.4404(11)$ & $\mathrm{N} 8-\mathrm{H} 4$ & $0.92(2)$ \\
\hline $\mathrm{N} 2-\mathrm{C} 3$ & $1.4486(10)$ & $\mathrm{C} 1-\mathrm{C} 3$ & $1.3817(11)$ \\
\hline $\mathrm{N} 3-\mathrm{C} 1$ & $1.3610(10)$ & $\mathrm{C} 2-\mathrm{H} 1$ & $0.92(2)$ \\
\hline $\mathrm{N} 3-\mathrm{C} 2$ & $1.3487(11)$ & $\mathrm{C} 4-\mathrm{C} 6$ & $1.3771(11)$ \\
\hline $\mathrm{N} 4-\mathrm{C} 2$ & $1.3282(10)$ & $\mathrm{C} 5-\mathrm{H} 3$ & $0.91(2)$ \\
\hline $\mathrm{O} 1-\mathrm{N} 1-\mathrm{O} 2$ & $125.12(8)$ & $\mathrm{C} 6-\mathrm{N} 8-\mathrm{H} 4$ & $125.6(14)$ \\
\hline $\mathrm{O} 1-\mathrm{N} 1-\mathrm{C} 1$ & $115.84(7)$ & $\mathrm{N} 1-\mathrm{C} 1-\mathrm{C} 3$ & $135.58(7)$ \\
\hline $\mathrm{O} 2-\mathrm{N} 1-\mathrm{C} 1$ & $119.01(7)$ & $\mathrm{N} 3-\mathrm{C} 1-\mathrm{C} 3$ & $105.73(7)$ \\
\hline $\mathrm{O} 3-\mathrm{N} 2-\mathrm{O} 4$ & $124.66(7)$ & $\mathrm{N} 1-\mathrm{C} 1-\mathrm{N} 3$ & $118.30(6)$ \\
\hline $\mathrm{O} 3-\mathrm{N} 2-\mathrm{C} 3$ & $118.67(7)$ & $\mathrm{N} 3-\mathrm{C} 2-\mathrm{N} 4$ & $111.94(7)$ \\
\hline $\mathrm{O} 4-\mathrm{N} 2-\mathrm{C} 3$ & $116.66(7)$ & $\mathrm{N} 2-\mathrm{C} 3-\mathrm{C} 1$ & $131.32(7)$ \\
\hline $\mathrm{C} 1-\mathrm{N} 3-\mathrm{C} 2$ & $106.95(7)$ & $\mathrm{N} 4-\mathrm{C} 3-\mathrm{C} 1$ & $110.21(6)$ \\
\hline $\mathrm{C} 2-\mathrm{N} 4-\mathrm{C} 3$ & $105.15(7)$ & $\mathrm{N} 2-\mathrm{C} 3-\mathrm{N} 4$ & $118.47(7)$ \\
\hline $\mathrm{C} 1-\mathrm{N} 3-\mathrm{H} 2$ & $127.2(15)$ & $\mathrm{N} 3-\mathrm{C} 2-\mathrm{H} 1$ & $121.3(15)$ \\
\hline $\mathrm{C} 2-\mathrm{N} 3-\mathrm{H} 2$ & $125.9(15)$ & $\mathrm{N} 4-\mathrm{C} 2-\mathrm{H} 1$ & $126.7(15)$ \\
\hline $\mathrm{O} 5-\mathrm{N} 5-\mathrm{C} 4$ & $117.25(7)$ & $\mathrm{N} 7-\mathrm{C} 4-\mathrm{C} 6$ & $110.60(7)$ \\
\hline $\mathrm{O} 6-\mathrm{N} 5-\mathrm{C} 4$ & $118.16(7)$ & $\mathrm{N} 5-\mathrm{C} 4-\mathrm{N} 7$ & $119.23(7)$ \\
\hline $\mathrm{O} 5-\mathrm{N} 5-\mathrm{O} 6$ & $124.55(8)$ & $\mathrm{N} 5-\mathrm{C} 4-\mathrm{C} 6$ & $130.13(7)$ \\
\hline $\mathrm{O} 8-\mathrm{N} 6-\mathrm{C} 6$ & $116.27(7)$ & $\mathrm{N} 7-\mathrm{C} 5-\mathrm{N} 8$ & $112.21(7)$ \\
\hline $\mathrm{O} 7-\mathrm{N} 6-\mathrm{C} 6$ & $118.28(7)$ & $\mathrm{N} 8-\mathrm{C} 6-\mathrm{C} 4$ & $105.81(6)$ \\
\hline $\mathrm{O} 7-\mathrm{N} 6-\mathrm{O} 8$ & $125.42(7)$ & $\mathrm{N} 6-\mathrm{C} 6-\mathrm{N} 8$ & $120.37(7)$ \\
\hline $\mathrm{C} 4-\mathrm{N} 7-\mathrm{C} 5$ & $104.72(7)$ & $\mathrm{N} 6-\mathrm{C} 6-\mathrm{C} 4$ & $133.38(7)$ \\
\hline $\mathrm{C} 5-\mathrm{N} 8-\mathrm{C} 6$ & $106.67(7)$ & $\mathrm{N} 7-\mathrm{C} 5-\mathrm{H} 3$ & $126.3(15)$ \\
\hline $\mathrm{C} 5-\mathrm{N} 8-\mathrm{H} 4$ & $127.5(14)$ & $\mathrm{N} 8-\mathrm{C} 5-\mathrm{H} 3$ & $121.5(15)$ \\
\hline $\mathrm{O} 1-\mathrm{N} 1-\mathrm{C} 1-\mathrm{N} 3$ & $-2.71(11)$ & $\mathrm{O} 7-\mathrm{N} 6-\mathrm{C} 6-\mathrm{N} 8$ & $159.07(7)$ \\
\hline $\mathrm{O} 1-\mathrm{N} 1-\mathrm{C} 1-\mathrm{C} 3$ & $-174.29(9)$ & $\mathrm{O} 7-\mathrm{N} 6-\mathrm{C} 6-\mathrm{C} 4$ & $-12.11(12)$ \\
\hline $\mathrm{O} 2-\mathrm{N} 1-\mathrm{C} 1-\mathrm{N} 3$ & $175.41(8)$ & $\mathrm{O} 8-\mathrm{N} 6-\mathrm{C} 6-\mathrm{N} 8$ & $-18.96(10)$ \\
\hline $\mathrm{O} 2-\mathrm{N} 1-\mathrm{C} 1-\mathrm{C} 3$ & $3.83(14)$ & $\mathrm{O} 8-\mathrm{N} 6-\mathrm{C} 6-\mathrm{C} 4$ & $169.86(8)$ \\
\hline $\mathrm{O} 3-\mathrm{N} 2-\mathrm{C} 3-\mathrm{N} 4$ & $163.63(7)$ & $\mathrm{C} 4-\mathrm{N} 7-\mathrm{C} 5-\mathrm{N} 8$ & $-0.32(9)$ \\
\hline $\mathrm{O} 3-\mathrm{N} 2-\mathrm{C} 3-\mathrm{C} 1$ & $-15.76(12)$ & $\mathrm{C} 5-\mathrm{N} 7-\mathrm{C} 4-\mathrm{N} 5$ & $-177.47(7)$ \\
\hline $\mathrm{O} 4-\mathrm{N} 2-\mathrm{C} 3-\mathrm{N} 4$ & $-15.16(11)$ & $\mathrm{C} 5-\mathrm{N} 7-\mathrm{C} 4-\mathrm{C} 6$ & $0.55(9)$ \\
\hline $\mathrm{O} 4-\mathrm{N} 2-\mathrm{C} 3-\mathrm{C} 1$ & $165.45(8)$ & $\mathrm{C} 5-\mathrm{N} 8-\mathrm{C} 6-\mathrm{N} 6$ & $-173.00(7)$ \\
\hline $\mathrm{C} 2-\mathrm{N} 3-\mathrm{C} 1-\mathrm{N} 1$ & $-174.24(7)$ & $\mathrm{C} 5-\mathrm{N} 8-\mathrm{C} 6-\mathrm{C} 4$ & $0.35(8)$ \\
\hline $\mathrm{C} 2-\mathrm{N} 3-\mathrm{C} 1-\mathrm{C} 3$ & $-0.35(8)$ & $\mathrm{C} 6-\mathrm{N} 8-\mathrm{C} 5-\mathrm{N} 7$ & $-0.02(9)$ \\
\hline $\mathrm{C} 1-\mathrm{N} 3-\mathrm{C} 2-\mathrm{N} 4$ & $1.06(9)$ & $\mathrm{N} 3-\mathrm{C} 1-\mathrm{C} 3-\mathrm{N} 4$ & $-0.44(9)$ \\
\hline $\mathrm{C} 3-\mathrm{N} 4-\mathrm{C} 2-\mathrm{N} 3$ & $-1.31(9)$ & $\mathrm{N} 1-\mathrm{C} 1-\mathrm{C} 3-\mathrm{N} 2$ & $-8.71(15)$ \\
\hline $\mathrm{C} 2-\mathrm{N} 4-\mathrm{C} 3-\mathrm{N} 2$ & $-178.45(7)$ & $\mathrm{N} 1-\mathrm{C} 1-\mathrm{C} 3-\mathrm{N} 4$ & $171.86(8)$ \\
\hline $\mathrm{C} 2-\mathrm{N} 4-\mathrm{C} 3-\mathrm{C} 1$ & $1.06(9)$ & $\mathrm{N} 3-\mathrm{C} 1-\mathrm{C} 3-\mathrm{N} 2$ & $178.98(8)$ \\
\hline $\mathrm{O} 6-\mathrm{N} 5-\mathrm{C} 4-\mathrm{C} 6$ & $-25.25(12)$ & $\mathrm{N} 5-\mathrm{C} 4-\mathrm{C} 6-\mathrm{N} 6$ & $-10.74(14)$ \\
\hline $\mathrm{O} 5-\mathrm{N} 5-\mathrm{C} 4-\mathrm{N} 7$ & $-25.48(11)$ & $\mathrm{N} 5-\mathrm{C} 4-\mathrm{C} 6-\mathrm{N} 8$ & $177.17(7)$ \\
\hline $\mathrm{O} 5-\mathrm{N} 5-\mathrm{C} 4-\mathrm{C} 6$ & $156.95(8)$ & $\mathrm{N} 7-\mathrm{C} 4-\mathrm{C} 6-\mathrm{N} 6$ & $171.53(8)$ \\
\hline $\mathrm{O} 6-\mathrm{N} 5-\mathrm{C} 4-\mathrm{N} 7$ & $152.33(7)$ & $\mathrm{N} 7-\mathrm{C} 4-\mathrm{C} 6-\mathrm{N} 8$ & $-0.57(8)$ \\
\hline
\end{tabular}


Hydrogen-bond geometry $\left(\AA,{ }^{\circ}\right)$

\begin{tabular}{lllll}
\hline$D-\mathrm{H} \cdots A$ & $D-\mathrm{H}$ & $\mathrm{H} \cdots A$ & $D \cdots A$ & $D-\mathrm{H} \cdots A$ \\
\hline $\mathrm{N} 3-\mathrm{H} 2 \cdots \mathrm{N} 7^{\mathrm{i}}$ & $0.90(2)$ & $1.96(2)$ & $2.836(1)$ & $163(2)$ \\
$\mathrm{N} 8-\mathrm{H} 4 \cdots \mathrm{N} 44^{\mathrm{ii}}$ & $0.92(2)$ & $1.89(2)$ & $2.807(1)$ & $179(3)$ \\
\hline
\end{tabular}

Symmetry codes: (i) $-x+1 / 2, y-1 / 2,-z+1 / 2$; (ii) $-x,-y+1,-z+1$. 